\title{
Gamma spectroscopy methodology for large amounts of environmental sam- ples in Sweden 30 years after the Chernobyl accident
}

\author{
Mattias Lantz ${ }^{1, *}$, Erik Andersson-Sundén ${ }^{1}$, Peter Andersson ${ }^{1}$, Abigail Barker ${ }^{2}$, Cecilia Gustavsson ${ }^{1}$, Anders \\ Hjalmarsson ${ }^{1}$, Marek Jacewicz ${ }^{1}$, Karl Lundén ${ }^{3}$, Pawel Marciniewski ${ }^{1}$, Stephan Pomp ${ }^{1}$, Vikram Rathore ${ }^{1}$, and Volker \\ Ziemann $^{1}$ \\ ${ }^{1}$ Department of Physics and Astronomy, Uppsala University, Sweden \\ ${ }^{2}$ Department of Earth Sciences, Uppsala University, Sweden \\ ${ }^{3}$ Department of Forest Mycology and Plant Pathology, Swedish Agricultural University, Sweden
}

\begin{abstract}
In a Swedish citizen science project, more than 200 elementary school classes participated in collecting fungi, soil samples, and droppings from deer and wild boar, from all over Sweden. The samples have been sent to a laboratory at Uppsala University where they are being analyzed through gamma spectroscopy with a shielded HPGe detector. The main objective is to scan the samples for ${ }^{137} \mathrm{Cs}$ from the Chernobyl accident and compare the data with measurements from 1986, but uptake of naturally occuring radionuclides like ${ }^{40} \mathrm{~K}$ and radon daughters will also be determined. Together with the soil samples, transfer factors will be derived, and correlations for these factors will be sought for different species of fungi and soil types. The potential for correlating the results with different biological processes will also be investigated, in part through the collected animal droppings. This is a work in progress where the present status of the experimental setup and methodology are presented. Issues with the initial approach for corrections are discussed and preliminary results are presented.
\end{abstract}

\section{Introduction}

The Chernobyl nuclear accident in 1986 distributed radioactive nuclides, primarily ${ }^{129 m, 132} \mathrm{Te},{ }^{131,133} \mathrm{I}$, ${ }^{134,136,137} \mathrm{Cs}$ and noble gases, over large areas of the former Soviet Union and Europe [1]. Sweden received about five percent ot the released ${ }^{137} \mathrm{Cs}$, which affected local reindeer herding, the foraging of wild berries and fungi from the forests, and fishing from lakes. Effects on agriculture was limited to the first few months and concerned mainly dairy products due to their affinity for uptake of radioactive iodine with half lives of eight days or shorter [2].

During the 33 years that have passed since the accident, the only remaining radionuclide of relevance is ${ }^{137} \mathrm{Cs}$ with a physical half-life of 30 years. Due to biological processes and depending on soil types the caesium may have been dispersed from where it was originally deposited, and it is therefore of interest to study such processes.

In general, the levels of ${ }^{137} \mathrm{Cs}$ in plants and animals is not a health risk for consumption, but the issue is still raising public concern.

For these reasons the citizen science project Strålande Jord (the name in Swedish has a double meaning in both words; "Brilliant/Irradiating Earth/Soil") was arranged in order to study the actual situation today in edible fungi (mushrooms). Upper elementary school classes (age 1315 years) in Sweden were invited to participate [3] and

*e-mail: mattias.lantz@physics.uu.se over 200 classes signed up for the project. The reason for reaching out to this age group was also to expose them to different aspects of natural sciences, with focus on radioactivity, fungi and scientific methodology. The project was arranged so that it would fit into the school curriculum for different subjects, and an instruction manual for the teachers gave suggestions for how to involve mathematics, natural and social sciences into the project [4].

\section{Data taking}

\subsection{Gathering of samples}

More detailed information about the citizen science aspects of the project is given elsewhere $[5,6]$, here a brief summary is given. The school classes were equipped with a toolkit for gathering fungi, soil samples from the same locations, and animal droppings from deer or boar, if found. The samples were gathered by the pupils during the time period august-november 2018. A list with 10 preferred species of edible fungi, all of them growing in most parts of Sweden, was given. A protocol for identified species, GPS positions and other information of relevance was to be filled in by the pupils. The fungi were then to be weighted, dried and ground into small pieces before being inserted into a plastic containers with $180 \mathrm{ml}$ volume and sent to Uppsala University together with the other samples. Each school class received containers for three different samples of fungi or animal droppings, and three related soil samples. 


\subsection{Gamma spectroscopy}

At Uppsala University the content of radionuclides in the fungi samples were measured with a lead-shielded Ortec GEM coaxial High Purity Germanium (HPGe) detector with $30 \%$ relative efficiency and $2.5 \mathrm{keV}$ energy resolution (FWHM) at $1.33 \mathrm{MeV}$. The intrinsic efficiency has been determined with standardized radioactive point sources with known activities.

For each measurement the plastic container was controlled for weight and filling height and then positioned directly on top of the HPGe detector in order to optimize the geometrical efficiency. Each sample was measured for at least 30 minutes in order to obtain sufficient statistics. Samples with very low count rates or small amounts of fungi were remeasured over nights and weekends. The gamma spectroscopy measurements were performed over a time period of three months, and background measurements were performed at regular intervals.

Ideally each plastic container should be completely filled, but it was quite common that they were only partly filled or contained very small amounts of fungi. A subset of such samples were after the first measurement transferred to smaller plastic containers in order to improve the geometrical efficiency, and then remeasured. Comparisons of measurements with both containers gave consistent results after corrections for the different geometries. Figure 1 shows two samples in the large plastic containers and one sample that has been transferred to the smaller container. As seen there is also a variation in granularity of the ground fungi samples.

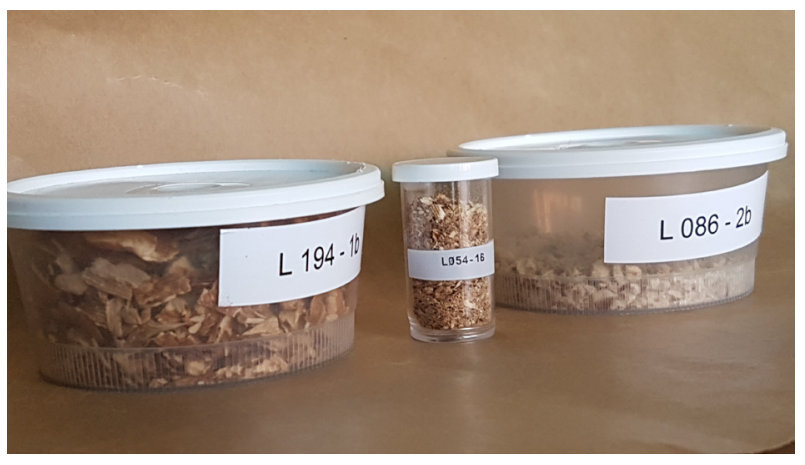

Figure 1. Plastic containers with samples of fungi, the contents in containers with small amounts of fungi were transferred to the smaller containers in order to improve the measurement conditions. The sticker on each container has the identification number for each sample.

\section{Analysis of data}

\subsection{Peak identification}

The pulse height spectra obtained from the gamma spectroscopy were initially analyzed with the Ortec Maestro MCA programme [7] that has been used for the data taking. Focus has been on ${ }^{137} \mathrm{Cs}$ and ${ }^{40} \mathrm{~K}$, but also radon daughters have have been searched for as the three elements caesium, potassium and radium are in the same chemical group and may therefore compete for the uptake by fungi.

\subsection{Corrections and remaining issues}

A plastic container filled with fungi is a radioactive source with a distributed geometry, and in order to correct for contributions from different parts of the container the full geometry has to be taken into account. Furthermore, as most containers were only partly filled, it was necessary also to correct for the amount of fungi in each container. As the fungi in general was ground and uniformly distributed within the container the geometrical correction was based on determining the height of the sample within each container.

The geometrical effects mentioned here has at a first stage been corrected for by creating efficiency curves from the results of simulations of the HPGe detector setup and the plastic containers. The Monte Carlo code GEANT4 [8] was used for these simulations and cross-checked independently with FLUKA [9], as expected the two codes gave equivalent results. Figure 2 shows the efficiency curves for ${ }^{137} \mathrm{Cs}$ and ${ }^{40} \mathrm{~K}$ at different sample heights in large and small containers. Also shown is simulated point sources placed at different heights, and a comparison with a measured point source of ${ }^{137} \mathrm{Cs}$. The error bars of the experimental source are dominated by the uncertainty of the source strength. As seen there is a discrepancy between the experiment and the simulation of the point source, the reason is most likely due to incorrect information about the internal geometry of the HPGe detector. Thus the simulated efficiency curves are too high and will result in a 10-15 percent systematic underestimate of the actual activities in each container.

Dried fungi are not very dense and as a first approximation it was not considered necessary to correct for selfshielding effects. This assumption is currently being investigated and may result in a small correction. The soil samples contain more dense materials and therefore it is necessary to determine correction functions for them.

The plastic containers are not completely flat in the bottom. A correction is therefore necessary as this is the region at closest distance to the HPGe detector crystal. Preliminary tests indicate that such corrections will increase the final results with about 5 percent.

Other corrections, such as true coincidence summing, have not been necessary as there is only one gamma transition each for ${ }^{137} \mathrm{Cs}$ and ${ }^{40} \mathrm{~K}$. No radon daughters have been observed in the fungi, but for the soil samples such elements are more likely to be found, and corrections may be necessary as several of the beta decays have multiple gamma transitions.

The determined activities are for dry weight. In order to compare the results with the governmental limit for ${ }^{137} \mathrm{Cs}$ of $1500 \mathrm{~Bq} / \mathrm{kg}$ for wet weight, a conversion factor of 0.08 was applied. This value was determined as the average value for all the given samples, as determined and recorded in the protocols by the school classes. As there are variations between different species individual correction factors are yet to be implemented. This will affect the 


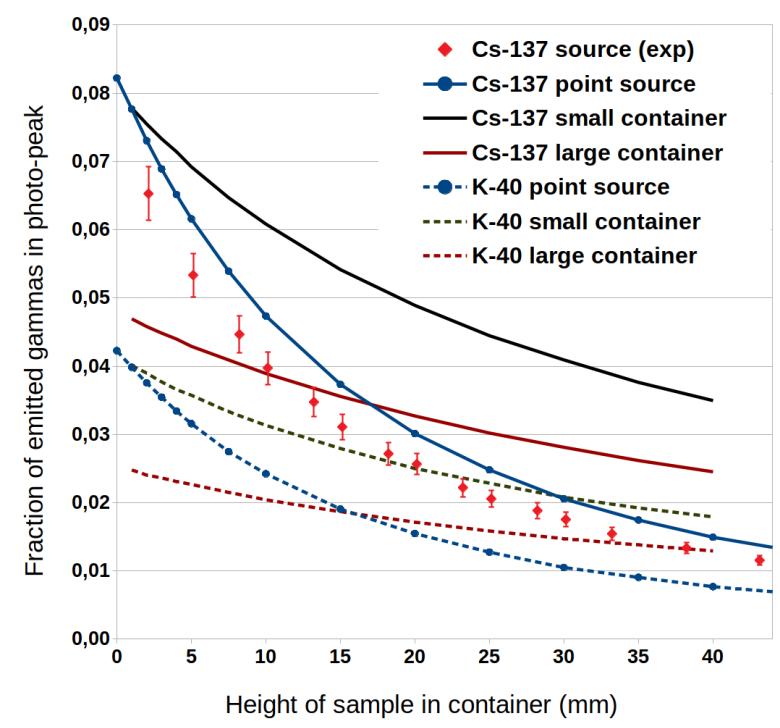

Figure 2. HPGe efficiency curves simulated with FLUKA for different heights of the samples in the small and large plastic containers, and point sources at different heights along the central axis. The simulated point source for ${ }^{137} \mathrm{Cs}$ is compared with an experimental point source, the discrepancy is discussed in the text.

final result somewhat for each individual sample and may in the end be the largest source of error.

Finally the results have been corrected for the gamma emission probabilities for ${ }^{137} \mathrm{Cs}(85.1 \%)$ and ${ }^{40} \mathrm{~K}$ $(10,66 \%)$.

In summary, there are several issues identified where our initial methods result in an underestimation the actual activity, the combined effect is about 20 percent. A few samples have been sent to other laboratories in order to cross-check our measurement method, preliminary results confirm the discrepancy of about 20 percent. Other reference sources will be used in order to account for the geometrical effects. We are also investigating the possibility to perform a tomographic examination of the internal parts of the HPGe detector.

\section{Preliminary results}

A total of 248 containers with fungi were obtained from 137 different school classes. Only two out of these samples had levels of ${ }^{137} \mathrm{Cs}$ above the governmental limit of $1500 \mathrm{~Bq} / \mathrm{kg}$. The data shows a broad distribution where most of the samples have activities that are also below the $300 \mathrm{~Bq} / \mathrm{kg}$ limit used in Sweden for staplefood [2]. The uptake of the naturally occurring radionuclide ${ }^{40} \mathrm{~K}$ is more uniform than for ${ }^{137} \mathrm{Cs}$, which is to be expected as potassium is a part of the composition of most fungi. Figure 3 shows a histogram for the distribution of the activities for ${ }^{137} \mathrm{Cs}$ and ${ }^{40} \mathrm{~K}$, the values on the $\mathrm{X}$-axis show the limits for each bin. Samples below the determined minimal detectable activity (MDA), are shown in the first bin. The MDA values vary for each sample as each sample had

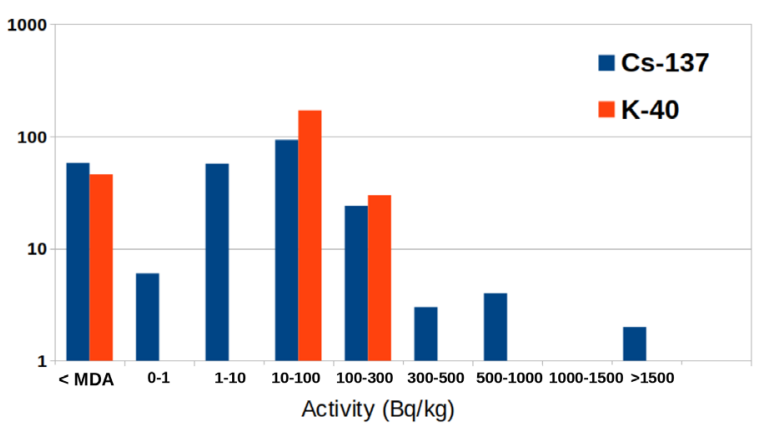

Figure 3. Distribution of preliminary results for ${ }^{137} \mathrm{Cs}$ and ${ }^{40} \mathrm{~K}$. Samples below the minimum detectable activity (MDA) are shown in the first column.

different mass and geometry, typical values were about $0.5 \mathrm{~Bq} / \mathrm{kg}$ for ${ }^{137} \mathrm{Cs}$ and about $30 \mathrm{~Bq} / \mathrm{kg}$ for ${ }^{40} \mathrm{~K}$.

In order to communicate the results back to the participating school classes, each sample was inserted on Google Maps with different visualizations. On the map each sample is shown as an icon, when clicking on the icon a window opens up with related information about the school class, GPS coordinates, species of fungus and measured activity. Figure 4 shows a screenshot of one of the Google Map visualizations where each sample is colour-coded according to its activity of ${ }^{137} \mathrm{Cs}[10]$. In the figure is also displayed a map from the Geological Survey of Sweden from 1986, displaying the distribution of ${ }^{137} \mathrm{Cs}$ in Sweden [11]. Both maps can be viewed with higher resolution by following the links in the references. The samples with the highest values tend to be in the areas that received most of the deposition from the Chernobyl accident. We did not recieve so many samples from the areas with most deposition from the Chernobyl accident, but overall the measurements display suprisingly low levels of ${ }^{137} \mathrm{Cs}$. Complementary samples are planned to be gathered at selected locations in order to investigate possible reasons for the low levels.

As this is work in progress the given results are approximate and will be subject to change as the different corrections used are being revised. It should be pointed out that the identified underestimation of the actual activities does not change the general conclusions that the levels of ${ }^{137} \mathrm{Cs}$ are much lower than expected.

\section{Conclusions and outlook}

The citizen science project Strålande Jord has been a rewarding experience but is not without challenges. The main source of uncertainty is from the information given by the school classes regarding the weights of the samples before and after drying, which we have very limited possibilities to verify. Overall we expect the information to be reliable, but in order to build confidence further collection of samples are being planned.

The large discrepancy in the corrections for geometrical effects is presently being addressed, and the determina- 


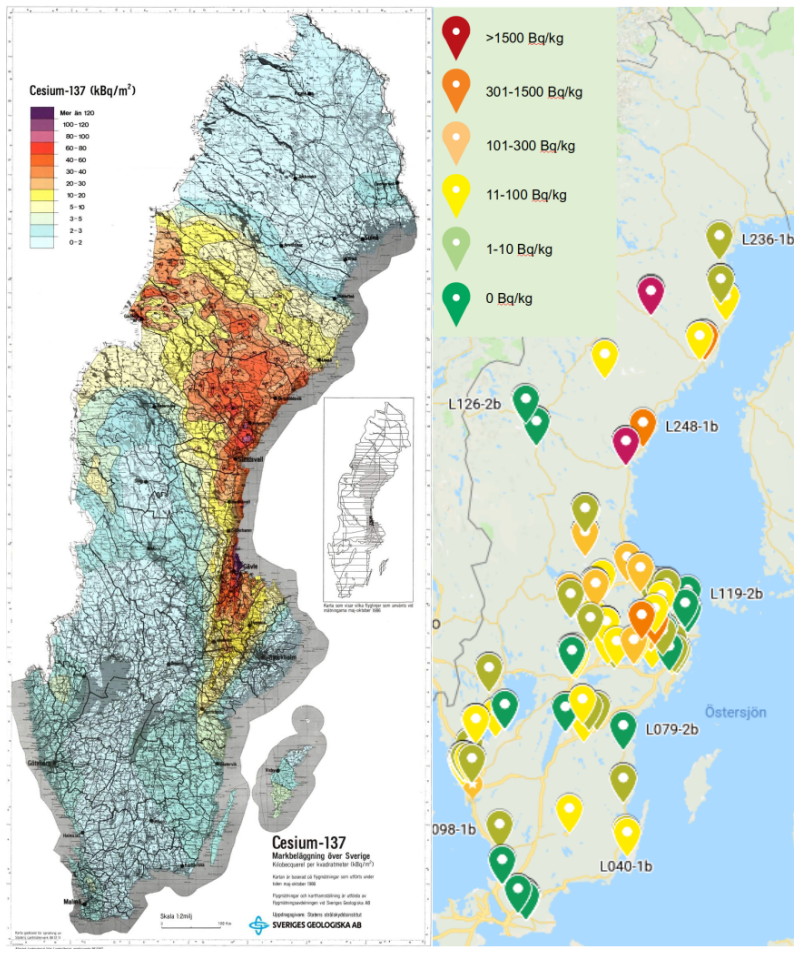

Figure 4. The results of the airborne measurements of ${ }^{137} \mathrm{Cs}$ in $\mathrm{kBq} / \mathrm{m}_{2}$, performed by the Geological Survey in Sweden in 1986 (left) [11], and a Google Maps visualization of the distribution of collected fungi samples in the citizen science project, colorcoded for activity in $\mathrm{Bq} / \mathrm{kg}$ (right) [10]. For better view, follow the links given in the references.

tion of the peaks will be cross-checked with an automated analysis programme written in Python [12].

Measurements on the related soil samples is ongoing and will be used in order to determine transfer factors for ${ }^{137} \mathrm{Cs}$ and ${ }^{40} \mathrm{~K}$ from the soils to the fungi. The data will be compared for different species of fungi, and the impact of different types of soil will also be investigated. There will also be an attempt to look for correlations from the about 50 samples of animal droppings that were gathered by the schools.

The main result from the project is that surprisingly few samples of fungi have ${ }^{137} \mathrm{Cs}$ activities above the governmental limit of $1500 \mathrm{~Bq} / \mathrm{kg}$. This is good news but raises new questions on why this is the case. Further measurements and comparisons with older data will be necessary.

This project has been supported and partly organized by the Unit for Communication and School Collaboration at the Faculty of Science and Technology, Uppsala University. Financial support has been received from Upptech, The Swedish Agricultural University, the Gothenburg Botanical Garden and the Swedish Radiation Safety Authority. The Geological Survey in Sweden has given permission for the use of the map of airborne measurements of ${ }^{137} \mathrm{Cs}$. The authors would also like to thank all the school teachers and pupils who have contributed with their time and effort.

\section{References}

[1] United Nations Scientific Committee on the Effects of Atomic Radiation, UNSCEAR 2008, Annex D: Health effects due to radiation from the Chernobyl accident (UNSCEAR, New York, 2011).

[2] J.O. Snihs, Contamination and Radiation Exposure, Evaluation and Measures in the Nordic Countries after the Chernobyl Accident, SSI report 1996:08 (1996).

[3] Web page for the citizen science project "Strålande Jord" web page, (2018). http://www.teknat.uu.se/ collaboration/school/mass-experiment/stralande-jord/

[4] Uppsala university, Teacher's manual, part 2, (2018). https://www.teknat.uu.se/collaboration/school/ mass-experiment/stralande-jord/lararhandledningl/

[5] C. Gustavsson et al., these procedings (2019).

[6] E. Andersson-Sundén et al., Nuclear Physics News 29(2), 25-28 (2019).

[7] Ortec, Maestro-32 MCA Emulator, (2008).

[8] S. Agostinelli et al., Nucl. Instrum. Methods A 506, 250 (2003).

[9] A. Ferrari et al., FLUKA: a multi-particle transport code, CERN-2005-10 (2005), INFN/TC_05/11, SLACR-773 (2005).

G. Battistoni et al., Annals of Nuclear Energy 82, 10-18 (2015).

[10] Google map visualization of the positions and results from all measurements in the project, (2019). https:// tinyurl.com/y8o5g76u

[11] Geological Survey of Sweden, Airborne gamma radiation measurements after the Chernobyl accident, (2019). https://www.sgu.se/en/aboutsgu/tasks-and-activities/geological-mappingsurveys/geophysical-mapping/airborne-geophysicalmeasurements/airborne-gamma-radiationmeasurements-after-the-chernobyl-accident/

[12] D. Nordman, BSc thesis work, Uppsala University (2018). http://uu.diva-portal.org/smash/record.jsf?pid= diva2\\%3A1235718\\&dswid=7436 